IJMMS 26:1 (2001) 1-5

PII. S0161171201020178

http://ijmms.hindawi.com

(C) Hindawi Publishing Corp.

\title{
SOME REMARKS ON THE SEVERI VARIETIES OF SURFACES IN $\mathbb{P}^{3}$
}

\section{CHEN}

(Received 13 December 2000)

\begin{abstract}
Continuing the work of Chiantini and Ciliberto (1999) on the Severi varieties of curves on surfaces in $\mathbb{P}^{3}$, we complete the proof of the existence of regular components for such varieties.
\end{abstract}

2000 Mathematics Subject Classification. 14H10, 14B07.

1. Introduction. Let $\mathscr{L}$ be a line bundle on a smooth surface $S$. Fix a positive integer $\delta$ and the Severi variety $V_{\mathscr{L}, \delta}^{0}$ is defined as the subscheme of $|\mathscr{L}|$ consisting of irreducible curves in $|\mathscr{L}|$ with $\delta$ nodes and no other singularities. This scheme, or sometimes, its closure $V_{\mathscr{L}, \delta}$ in $|\mathscr{L}|$ is called the Severi variety of curves with $\delta$ nodes in $|\mathscr{L}|$. The Severi variety $V_{\mathscr{L}, \delta}^{0}$, if not empty, has the dimension at least $\max \{\operatorname{dim}|\mathscr{L}|-\delta,-1\}$, where $\max \{\operatorname{dim}|\mathscr{L}|-\delta,-1\}$ is called the expected dimension of $V_{\mathscr{L}, \delta}^{0}$. A reduced component of $V_{\mathscr{S}, \delta}^{0}$ that has the expected dimension is called a regular component of $V_{\mathscr{L}, \delta}^{0}$.

The Severi varieties of plane curves have been classically extensively studied. It is natural to study the Severi varieties on other surfaces. The Severi varieties of surfaces in $\mathbb{P}^{3}$ were studied in [2]. Let $S$ be a smooth surface of degree $d$ in $\mathbb{P}^{3}$ and let $\left|\mathrm{O}_{S}(n)\right|$ be the linear series on $S$ cut out by the degree $n$ surfaces in $\left|P^{3}\right|$. We use the notation $V_{n, \delta}^{0}(S)$ to denote the Severi variety of curves with $\delta$ nodes in $\left|\mathcal{O}_{S}(n)\right|$. It was proved in [2] that for $S$ general, $n \geq d$ and all $\delta$ with $0 \leq \delta \leq \operatorname{dim}\left(\left|O_{S}(n)\right|\right), V_{n, \delta}^{0}(S)$ has at least one component which is reduced of the expected dimension. That, combined with a construction of irregular components of $V_{n, \delta}^{0}(S)$ for $n \gg d \geq 8$ and some $\delta$, gives examples of reducible Severi varieties on surfaces in $\mathbb{P}^{3}$.

The purpose of this paper is to prove the main theorem in [2] in the case $n<d$, which was not covered in that paper. Namely, we show the following theorem.

THEOREM 1.1. For a general surface $S$ of degree $d$ in $\mathbb{P}^{3}, n<d$ and all $\delta$ with $0 \leq \delta \leq \operatorname{dim}\left(\left|O_{S}(n)\right|\right)=\left(\begin{array}{c}n+3 \\ 3\end{array}\right)-1$, the Severi variety $V_{n, \delta}^{0}(S)$ has at least one regular component.

\section{Proof of Theorem 1.1}

2.1. Sketch of the proof. Our construction of the regular component of $V_{n, \delta}^{0}(S)$ for $n<d$ is very close to that of Chiantini and Ciliberto in spirit: we take a degeneration of degree $d$ surfaces in $\mathbb{P}^{3}$ and try to locate a subscheme of $\left|O_{S_{0}}(n)\right|$ on the "degenerated" degree $d$ surface $S_{0}$ which is the limit of some components of the Severi varieties on the general fibers; we are done as long as the subscheme we find has the 
expected dimension of the Severi varieties on the general fibers. However, both the way we degenerate the surfaces and the way we locate those "limiting nodal curves" are different from those used in [2].

Let $X \subset \mathbb{P}^{3} \times \Delta$ be a pencil of surfaces of degree $d$ in $\mathbb{P}^{3}$ whose central fiber $X_{0}=$ $H_{1} \cup H_{2} \cup \cdots \cup H_{d}$ is a union of $d$ hyperplanes. Suppose that $H_{1}, H_{2}, \ldots, H_{d}$ are in relatively general position, so no four of them meet at a point. Suppose that the pencil $X$ is chosen to be general so that it has $d\left(\begin{array}{l}d \\ 2\end{array}\right)$ distinct base points and none of them is the intersection of three planes among $H_{1}, H_{2}, \ldots, H_{d}$. Then the threefold $X$ has exactly $d\left(\begin{array}{l}d \\ 2\end{array}\right)$ rational double points, which are the base points of the pencil, and no other singularities. These double points lie on the lines $L_{i j}=H_{i} \cap H_{j}$ for $i \neq j$ and $1 \leq i$, $j \leq d$.

There are various ways we may take the limit of the linear series $\left|\mathscr{O}_{X_{t}}(n)\right|$ as $t \rightarrow 0$. Here we just let $\lim _{t \rightarrow 0}\left|\mathcal{O}_{X_{t}}(n)\right|=\left|\mathcal{O}_{X_{0}}(n)\right|$. Let $C=\cup_{i=1}^{d} C_{i}$ be a curve in $\left|\mathcal{O}_{X_{0}}(n)\right|$, where $C_{i} \subset H_{i}$ for $i=1,2, \ldots, d$. If $C$ passes through a double point $p$ of $X$ on the line $L_{i j}$, and $C_{i}$ and $C_{j}$ meet $L_{i j}$ transversely at $p$ on $H_{i}$ and $H_{j}$, respectively, then $C$ can be deformed to a curve $C_{t} \in\left|\mathrm{O}_{X_{t}}(n)\right|$ with $p$ deforming to a node of $C_{t}$ (cf. [1, Theorem 2.2]). So, to produce $\delta$ nodes of $C_{t}$, it suffices to let $C$ pass through $\delta$ double points of $X$. Notice that we have enough double points to play with since $\delta \leq\left(\begin{array}{c}n+3 \\ 3\end{array}\right)-1 \leq d\left(\begin{array}{l}d \\ 2\end{array}\right)$ for $d \geq 4$ and $d>n$. So pick a collection $Z$ of $\delta$ double points of $X$ and there always exists $C \in\left|O_{X_{0}}(n)\right|$ passing through $Z$. But the catch here is that we have to make sure of the following things.

(1) Most important of all, we have to choose $Z$ such that $Z$ imposes independent conditions on $\left|O_{X_{0}}(n)\right|$, or equivalently, on $\left|\mathcal{O}_{\mathbb{p} 3}(n)\right|$.

(2) A general member $C \in\left|O_{X_{0}}(n)\right|$ that passes through $Z$ must be cut out by an irreducible surface $S$ of degree $n$ in $\mathbb{P}^{3}$.

(3) For a general member $C=\cup_{i=1}^{d} C_{i} \in\left|O_{X_{0}}(n)\right|$ that passes through $Z$, each $C_{i}$ must meet $L_{i j}$ transversely at points in $Z \cap L_{i j}$.

We are not sure whether part (3) is essential. It is conceivable that even if $C_{i}$ fails to meet $L_{i j}$ transversely at a point $p \in Z \cap L_{i j}$, it is still possible to deform $C$ to $C_{t}$ with $p$ becoming a node of $C_{t}$. However, we will verify (3) anyway since it is quite trivial by our construction.

2.2. Proof of Theorem 1.1 when $n<d-1$. First, we look at when $Z \cap H_{i}$ imposes independent conditions on $\left|\mathrm{O}_{H_{i}}(n)\right|$. In general, we may ask the following question.

QUESTION 2.1. Let $L_{1}, L_{2}, \ldots, L_{p}$ be $p$ lines on $\mathbb{P}^{2}$ and let $Z$ be a zero-dimensional subscheme of $\mathbb{P}^{2}$ which consists of $a_{1}$ points on $L_{1}, a_{2}$ points on $L_{2}, \ldots$, and $a_{p}$ points on $L_{p}$ with none of these points being the intersection of two lines among $L_{1}, L_{2}, \ldots, L_{p}$. Fix $n<p$, we ask that under what conditions does $Z$ impose independent conditions on the linear series $|\mathcal{O}(n)|$ ?

To be more specific, if $Z$ imposes independent conditions on $|\mathcal{O}(n)|$, then

(1) What kind of numerical conditions should be satisfied by $a_{1}, a_{2}, \ldots, a_{p}$ ?

(2) Should $L_{1}, L_{2}, \ldots, L_{p}$ be in general position or could they be arbitrary?

(3) Should the points $Z \cap L_{k}$ be in general position on $L_{k}$ or could they be arbitrary for $k=1,2, \ldots, p$ ? 
For example, it is not hard to obtain a necessary numerical condition on $a_{1}, a_{2}, \ldots, a_{p}$ by noticing that the linear series cut out by $|\mathcal{O}(n)|$ on a union of $k$ lines has dimension $n k-\left(k^{2}-3 k\right) / 2-1$ for $k \leq n$. So $a_{1}, a_{2}, \ldots, a_{p}$ have to satisfy the following condition.

For each $k \leq n$ and each $I \subset\{1,2, \ldots, p\}$ with $\# I=k$,

$$
\sum_{i \in I} a_{i} \leq n k-\frac{k^{2}-3 k}{2}-1 \text {. }
$$

Although we do not have the answer for Question 2.1 in general, we can prove a very special case which suffices for our purpose.

LEMMA 2.2. Let $L_{1}, L_{2}, \ldots, L_{n+1}$ be $n+1$ distinct lines on $\mathbb{P}^{2}$ and let $Z$ be a zerodimensional subscheme of $\mathbb{P}^{2}$ which consists of $a_{1}$ points on $L_{1}, a_{2}$ points on $L_{2}, \ldots$, and $a_{n+1}$ points on $L_{n+1}$ with none of these points being the intersection of two lines among $L_{1}, L_{2}, \ldots, L_{n+1}$. Suppose that $a_{k} \leq n+2-k$ for $k=1,2, \ldots, n+1$. Then $Z$ imposes independent conditions on $|\mathcal{O}(n)|$, that is, $H^{1}\left(I_{Z}(n)\right)=0$.

Proof. It suffices to show that there is no curve in $|\mathcal{O}(n)|$ passing through $Z$ if $a_{k}=n+2-k$ for $k=1,2, \ldots, n+1$.

Suppose that $a_{k}=n+2-k$ for $k=1,2, \ldots, n+1$ and there exists a curve $C$ of degree $n$ passing through $Z$. Since $C$ passes through $n+1$ points on $L_{1}$ and $\operatorname{deg} C=n, L_{1} \subset C$. Let $C=L_{1} \cup C^{\prime}$. Now $C^{\prime}$ passes through $n$ points on $L_{2}$ and $\operatorname{deg} C^{\prime}=n-1$. So $L_{2} \subset C$. So inductively we have $L_{k} \subset C$ for $k=1,2, \ldots, n+1$. Obviously, this is impossible because $\operatorname{deg} C=n$.

LEMMA 2.3. Let $H_{1}, H_{2}, \ldots, H_{n+2}$ be $n+2$ distinct planes in $\mathbb{P}^{3}$ with no three of them meeting along a line and let $L_{i j}=H_{i} \cap H_{j}$ for $1 \leq i<j \leq n+2$. Let $Z$ be a zerodimensional subscheme of $\mathbb{P}^{3}$ which consists of $a_{i j}$ points on $L_{i j}$ for $1 \leq i<j \leq n+2$. Suppose that $a_{i j} \leq n+3-j$ for $1 \leq i<j \leq n+2$. Then $Z$ imposes independent conditions on $|\mathbb{O}(n)|$.

Proof. It suffices to show that there are no surfaces in $|\mathcal{O}(n)|$ passing through $Z$ if $a_{i j}=n+3-j$ for $1 \leq i<j \leq n+2$.

Suppose that $a_{i j}=n+3-j$ for $1 \leq i<j \leq n+2$ and there is a degree $n$ surface $S$ in $\mathbb{P}^{3}$ passing through $Z$. First, we claim that $H_{1} \subset S$. If not, $C=S \cap H_{1}$ is a degree $n$ curve in $H_{1}$ passing through $Z \cap H_{1}$. Notice that $Z \cap H_{1}$ is a zero-dimensional subscheme of $H_{1}$ consisting of $n+1$ points on $L_{12}, n$ points on $L_{13}, \ldots$, and 1 point on $L_{1, n+2}$. So by Lemma 2.2, there is no degree $n$ curve passing through $Z \cap H_{1}$. Contradiction. So $H_{1} \subset S$ and let $S=H_{1} \cup S^{\prime}$. Next, we claim that $H_{2} \subset S^{\prime}$. If not, $C^{\prime}=S^{\prime} \cap H_{2}$ is a degree $n-1$ curve passing through $\left(Z \cap H_{2}\right) \backslash L_{12}$. Notice that $\left(Z \cap H_{2}\right) \backslash L_{12}$ is a zerodimensional subscheme of $H_{2}$ consisting of $n$ points on $L_{23}, n-1$ points on $L_{24}, \ldots$, and 1 point on $L_{2, n+2}$. So by Lemma 2.2, there is no degree $n-1$ curve passing through $\left(Z \cap H_{2}\right) \backslash L_{12}$. Contradiction. So we may carry on this line of argument and finally conclude that $H_{1} \cup H_{2} \cup \cdots \cup H_{n+1} \subset S$. This is impossible since $\operatorname{deg} S=n$.

Now, we go back to the proof of Theorem 1.1 in the case of $n<d-1$. It suffices to prove Theorem 1.1 when $\delta=\left({ }^{n+3}\right)-1$. As in the sketch of the proof, we need to find a collection $Z$ of $\delta$ double points of the family $X$ such that $Z$ imposes independent conditions on $\left|\mathcal{O}_{\mathbb{P} 3}(n)\right|$. 
The family $X$ has $d\left(\begin{array}{l}d \\ 2\end{array}\right)$ double points with $d$ points on each $L_{i j}$. Let $Z^{\prime}$ be a collection of $\left(\begin{array}{c}n+3 \\ 3\end{array}\right)-1$ double points of $X$ with exactly $n+3-j$ on each $L_{i j}$ for $1 \leq i<j \leq n+2$, and let $Z$ be a subset of $Z^{\prime}$ with one point removed on $L_{12}$.

By Lemma 2.3, $Z$ imposes $\delta$ independent conditions on $\left|\mathscr{O}_{\mathbb{P} 3}(n)\right|$. So $\operatorname{dim}\left|I_{Z}(n)\right|=0$. Of course, $\left|I_{Z}(n)\right|$ consists of exactly one surface $S$.

Next, we need to verify that $S$ is irreducible for a general choice of $X$. Choose an irreducible surface $S \in\left|\mathbb{O}_{\mathbb{p} 3}(n)\right|$ and a surface $S^{\prime} \in\left|\mathbb{O}_{\mathbb{p}^{3}}(d-n)\right|$. Let $X$ be the pencil of degree $d$ surfaces given by

$$
F_{1} F_{2} \cdots F_{d}+t G G^{\prime}=0
$$

where $F_{1}, F_{2}, \ldots, F_{d}$ are the defining equations of $H_{1}, H_{2}, \ldots, H_{d}$ and $G$ and $G^{\prime}$ are the defining equations of $S$ and $S^{\prime}$. Obviously, we may choose $Z$ to consist of points in $S \cap L_{i j}$ for $1 \leq i<j \leq n+2$. Then $S \in\left|I_{Z}(n)\right|$ and it is irreducible.

Finally, we need to verify that $S$ meets $L_{i j}$ transversely at points in $\left(Z \cap L_{i j}\right)$ for a general choice of $X$, which is quite obvious with the construction of $X$ in (2.2). This concludes the proof of Theorem 1.1 in the case of $n<d-1$.

2.3. Proof of Theorem 1.1 when $n=d-1$. The proof of Theorem 1.1 in the case of $n=d-1$ is a little more involved. Again it suffices to prove the theorem when $\delta=\left(\begin{array}{c}d+2 \\ 3\end{array}\right)-1$.

Let $Z^{\prime}$ be a collection of $\left({ }_{3}^{d+2}\right)-d$ double points of $X$ with exactly $d+2-j$ on each $L_{i j}$ for $1 \leq i<j \leq d$ and let $Z$ be a subset of $Z^{\prime}$ with one point removed on $L_{12}$.

By Lemma 2.3, $Z$ imposes independent conditions on $\left|\mathscr{O}_{\mathbb{P}^{3}}(d-1)\right|$. So $\operatorname{dim} \mid I_{Z}(d-$ $1) \mid=d$. Actually, we can very explicitly write down a basis for $H^{0}\left(I_{Z}(d-1)\right)$ as follows.

Since $Z^{\prime}$ also imposes independent conditions on $\left|\mathcal{O}_{\mathbb{P} 3}(d-1)\right|$, there exists $G \in$ $H^{0}\left(I_{Z}(d-1)\right)$ such that the surface defined by $G=0$ does not contain the line $L_{12}$. On the other hand, for each $1 \leq i \leq d, \prod_{j \neq i} F_{j}$ belongs to $H^{0}\left(I_{Z}(d-1)\right)$, where $F_{1}, F_{2}, \ldots, F_{d}$ are the defining equations of $H_{1}, H_{2}, \ldots, H_{d}$, respectively. It is easy to see that $G$ and $\prod_{j \neq i} F_{j}(i=1,2, \ldots, d)$ are linearly independent and hence they span the vector space $H^{0}\left(I_{Z}(d-1)\right)$.

So the restriction $\left|I_{Z \cap H_{i}} \otimes \mathcal{O}_{H_{i}}(d-1)\right|$ of $\left|I_{Z}(d-1)\right|$ to $H_{i}$ is a pencil of degree $d-1$ curves generated by $\left.G\right|_{H_{i}}$ and $\left.\left(\prod_{j \neq i} F_{j}\right)\right|_{H_{i}}$. By the same argument as before, we can show that $G$ defines a general surface of degree $d-1$ for a general choice of $X$. So the pencil $\left|I_{Z \cap H_{i}} \otimes \mathcal{O}_{H_{i}}(d-1)\right|$ contains an isolated irreducible curve $C_{i}$ with exactly one node. So $C=\cup_{i=1}^{d} C_{i} \in\left|O_{X_{0}}(d-1)\right|$ has $d$ nodes, passes through $\left(\begin{array}{c}d_{3}^{+1} \\ 3\end{array}\right)-d-1$ double points of $X$, and is isolated and cut out by an irreducible surface of degree $d-1$. Therefore, $C$ is the flat limit of an isolated irreducible curve on $X_{t}$ with exactly $\delta=\left(\begin{array}{c}d+2 \\ 3\end{array}\right)-1$ nodes. This concludes the proof of Theorem 1.1 when $n=d-1$.

ACKNOWLEDGEMENT. I would like to thank Luca Chiantini, who kindly explained to me in details his joint work with C. Ciliberto on the problem.

\section{REFERENCES}

[1] X. Chen, Rational curves on K3 surfaces, J. Algebraic Geom. 8 (1999), no. 2, 245-278, also preprint http://arxiv.org/abs/math.AG/9804075. MR 2000d:14057. Zbl 940.14024. 
[2] L. Chiantini and C. Ciliberto, On the Severi varieties of surfaces in $\mathbb{P}^{3}$, J. Algebraic Geom. 8 (1999), no. 1, 67-83, also preprint http://arxiv.org/abs/math.AG/9802009. MR 2000f:14082. Zbl 990.61182.

Xi Chen: Department Of Mathematics, South Hall, Room 6607, University OF CALIFORNIA, SANTA BARBARA, CA 93106, USA

E-mail address: xichen@math .ucsb.edu 


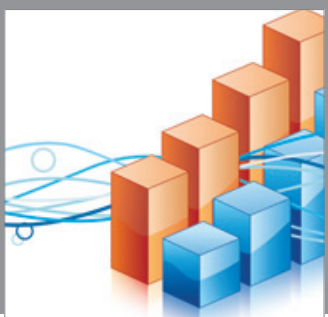

Advances in

Operations Research

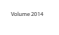

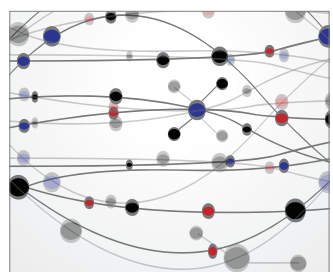

\section{The Scientific} World Journal
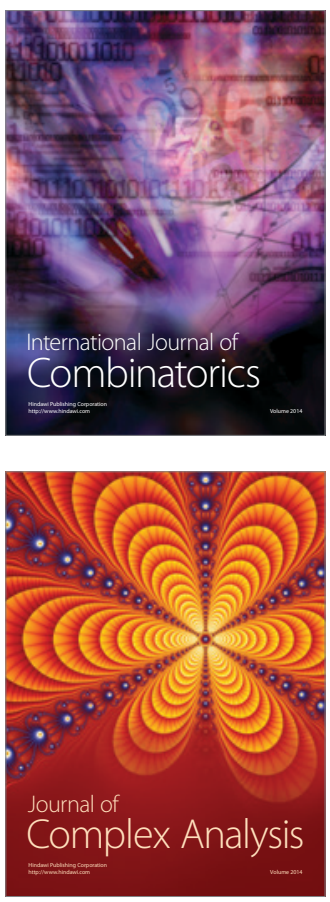

International Journal of

Mathematics and

Mathematical

Sciences
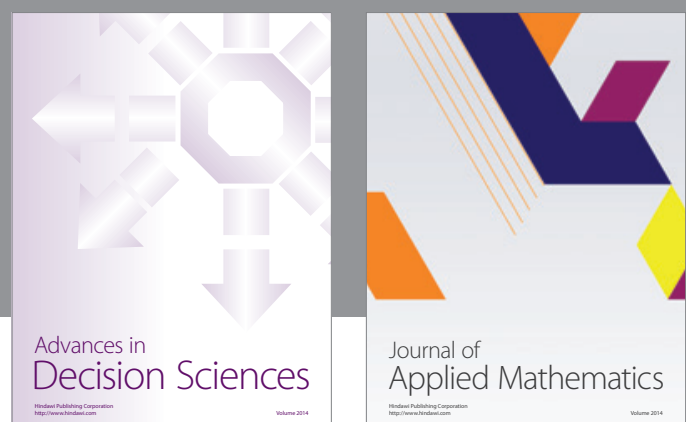

Journal of

Applied Mathematics
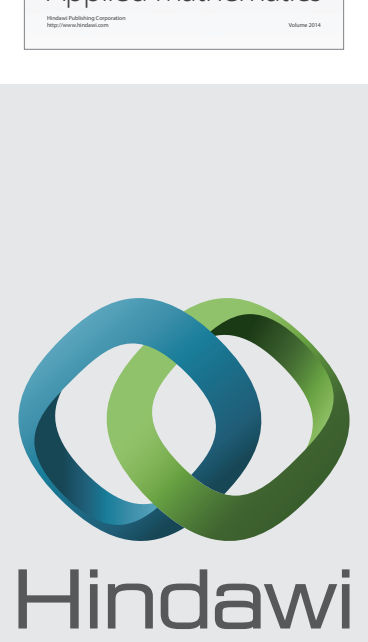

Submit your manuscripts at http://www.hindawi.com
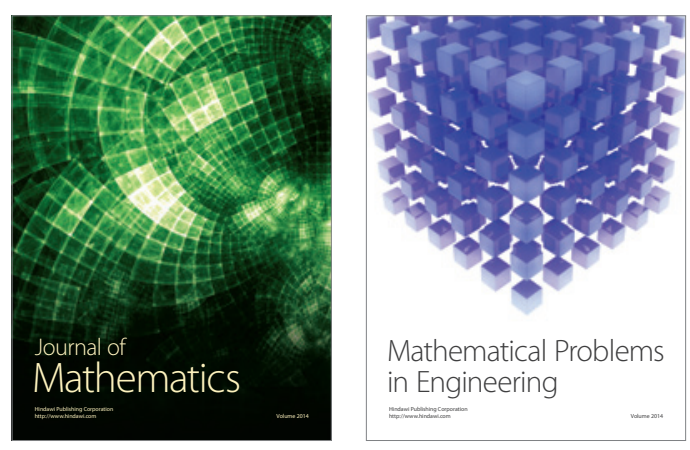

Mathematical Problems in Engineering
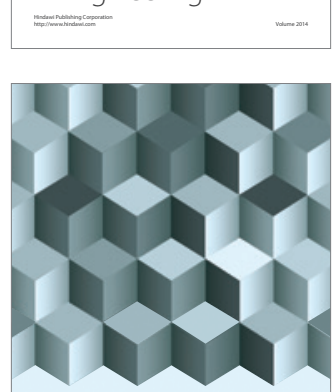

Journal of

Function Spaces
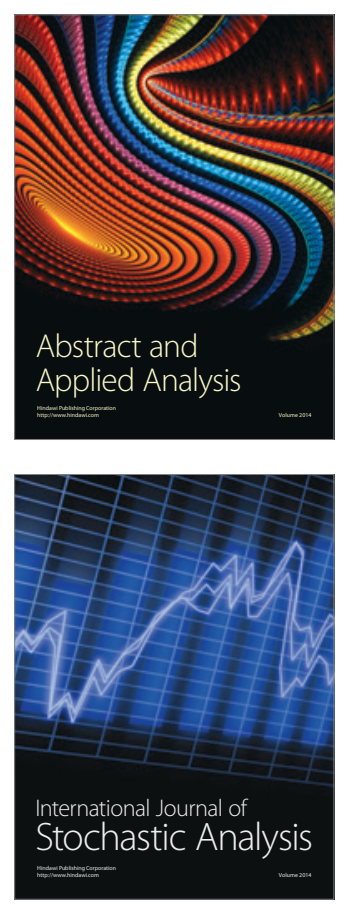

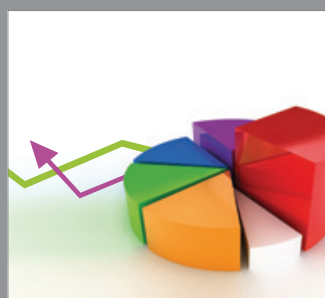

ournal of

Probability and Statistics

Promensencen
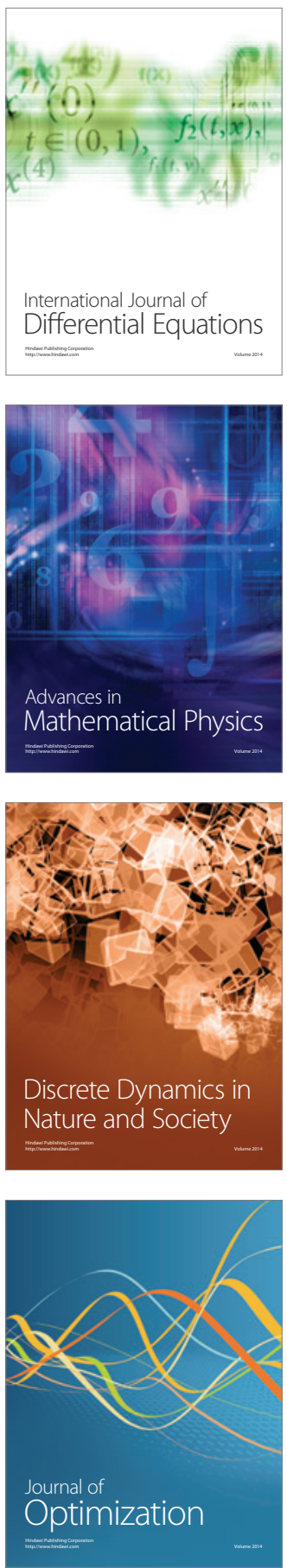